ISSN 0103-5150

Fisioter. Mov., Curitiba, v. 24, n. 4, p. 721-728, out./dez. 2011 Licenciado sob uma Licença Creative Commons

\title{
Avaliação postural de nadadores e sua relação com o tipo de respiração
}

\author{
Postural evaluation of swimmers and its \\ relation to type of breathing
}

\section{Gustavo Antonio Meliscki ${ }^{[a]}$, Luciana Zaranza Monteiro ${ }^{[b]}$, Carlos Alberto Giglio ${ }^{[c]}$}

[a] Graduando do curso de Fisioterapia pela Universidade de Ribeirão Preto (Unaerp), Ribeirão Preto, SP - Brasil, e-mail: gustavo.meliscki@gmail.com

[b] Doutoranda do Departamento de Clínica Médica da Faculdade de Medicina de Ribeirão Preto da Universidade de São Paulo (FMRP-USP), Ribeirão Preto, SP - Brasil, e-mail: lucianazaranza@usp.br

[c] Fisioterapeuta, docente do curso de Fisioterapia da Unaerp, Ribeirão Preto, SP - Brasil, e-mail: carlos.giglio@yahoo.com.br

\section{Resumo}

Introdução: A avaliação postural é importante em qualquer exame físico, tanto na reabilitação como na prevenção, e com ela poderemos adquirir condições de mudar hábitos inadequados ou ineficientes de cada indivíduo. Objetivo: Avaliar e comparar os gêneros das alterações posturais dos atletas de natação e verificar a associação dessas alterações com o tipo de respiração. Metodologia: Participaram 60 atletas, sendo que todos preencheram uma ficha, incluindo: sexo, idade, principal estilo do nado, tempo de prática do esporte e tipo de respiração predominante. Na avaliação postural, utilizou-se uma câmera fotográfica digital Sony

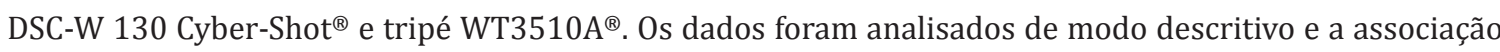
entre as variáveis utilizou o Teste Exato de Fisher, por meio do software SAS ${ }^{\circledR}$ 9. Resultados: 0 crawl foi o estilo de nado predominante entre os atletas; notamos que os homens (49\%) tinham respiração unilateral à direita; e as mulheres (41\%), à esquerda. Os homens (58\%) apresentaram mais hiperlordose lombar e as mulheres (31\%) escoliose convexa à direita $(p=0,01)$. Foi apresentada associação entre alterações posturais e o tipo de respiração no sexo masculino, sendo que aqueles que respiravam para o lado direito tinham ombro esquerdo elevado e escoliose convexa à esquerda $(p=0,02)$. Conclusão: Os resultados revelaram que os atletas que praticam respiração unilateral à direita apresentavam mais alterações posturais. Por ou- 
tro lado, a insuficiência de trabalhos publicados sobre a avaliação postural associado ao tipo de respiração de nadadores reforça a necessidade de novos estudos.

Palavras-chave: Avaliação. Postura. Natação. Respiração.

\section{Abstract}

Introduction: Postural evaluation is important in any physical examination regarding both rehabilitation and prevention, with the possibility of acquiring conditions that will permit changing the inadequate or inefficient habits of any individual. Objective: To evaluate and compare between genders the postural changes of swimming athletes and to determine the association of these changes with type of breathing. Methodology: Sixty athletes participated in the study, all of them filling out a chart with data about sex, age, main swimming style, time of practice of the sport and predominant type of breathing. A Sony DSC-W 130 Cyber-Shot ${ }^{\text {TM }}$ camera and a WT3510A $A^{\text {TM }}$ tripod were used for postural evaluation. The data were analyzed in a descriptive manner and the association between variables was determined by the Fisher exact test using the SAS ${ }^{\text {TM }} 9$ software. Results: The crawl swimming style was the predominant one among the athletes, with men (49\%) practicing unilateral breathing on the right and women (41\%) on the left. Men (58\%) presented more lumbar hyperlordosis and women (31\%) right convex scoliosis ( $p=0.01$ ). there was an association between postural changes and type of breathing among males, with those who breathed on the right side having elevation of the left shoulder and convex scoliosis on the left $(p=0.02)$. Conclusion: The results revealed that athletes who breathe unilaterally on the right have more postural alterations. However, in view of the scarcity of published studies on postural evaluation associated with type of breathing in swimmers, new studies are needed.

Keywords: Assessment. Posture. Swimming. Breathing.

\section{Introdução}

A natação pode ser considerada uma das habilidades motoras que podem ter ajudado o homem na sua luta evolutiva (1). Seus benefícios são muitos, evidentes e inquestionáveis, pode ser indicada como medida terapêutica que visa à cura ou coadjuvação no tratamento de alguma patologia, ou profilática que visa prevenir o surgimento de alguma doença (2).

No entanto, a natação de competição pode comprometer a saúde dos seus praticantes, exigindo tempo de dedicação considerável. Um nadador que treina de 20 a 30 horas por semana, por exemplo, executa mais de 500.000 ciclos de braçadas após o período de um ano (3). Essa grande repetição de movimentos, associada ao tipo de respiração adotada pelo atleta e adicionada à crescente falta de equilíbrio muscular, pode levá-lo a adotar algumas alterações posturais (3).

A respiração do nadador é específica. A expiração torna-se ativa e a inspiração, reflexa (4). 0 controle da respiração é vital para um bom desempenho na natação, exigindo precisão e ritmo (5-7). 0 tempo da inspiração é muito curto e deve ser realizado pela boca antes de imergir a cabeça na água. A expiração deve ser realizada de modo prolongado pela boca ou pela boca e nariz, concomitantemente, dentro da água $(5,6)$. A respiração bilateral é a ideal, pois ajuda a evitar a tensão muscular, oferecendo à musculatura do ombro e pescoço um descanso maior durante o tempo de apneia (8).

A postura corporal pode ser definida como a posição que o corpo adota no espaço e a relação direta de suas partes com a linha do centro de gravidade (1). A postura correta e o alinhamento esquelético ideal é aquele em que uma quantidade mínima de estresse e um mínimo esforço conduzem a eficiência máxima do corpo (9). A avaliação postural é uma importante ferramenta para que possamos determinar os desequilíbrios e adequar a melhor postura a cada indivíduo.

Há quase três décadas já se conhecem o diagnóstico, a prevenção e o tratamento das principais lesões na natação (10). Porém, o conhecimento das alterações posturais decorrentes da natação competitiva apresenta, ainda, muitas dúvidas, o que acarreta a necessidade de conhecimento aprofundado de sua fisiopatologia.

Assim, o objetivo deste trabalho foi avaliar e comparar entre os gêneros as alterações posturais dos 
atletas de natação e verificar a associação dessas alterações com o tipo de respiração.

\section{Materiais e métodos}

Foi realizado um estudo transversal com a equipe de elite da natação da Universidade de Ribeirão Preto (Unaerp - SP) no primeiro semestre de 2010. Foram selecionados 60 atletas de acordo com os critérios: voluntários física e mentalmente independentes, pertencente à faixa etária de 13 a 28 anos, ambos os sexos, com um tempo mínimo de prática da natação de três anos. Todos os 60 atletas aceitaram participar da pesquisa.

0 projeto de pesquisa foi aprovado pelo Comitê de Ética em Pesquisa em Seres Humanos (CEP) da Unaerp (número 031/2010). A autorização dos participantes foi realizada por meio do Termo de Consentimento Livre e Esclarecido, de acordo com a resolução CNS 196/96, em que os voluntários tiveram o direito a participar ou não do estudo, podendo interromper a pesquisa em qualquer momento. Para a participação na pesquisa para os menores de 18 anos, foi exigida também a assinatura dos pais ou responsável.

Para a coleta de dados, utilizamos uma ficha de identificação dos atletas (nome, sexo, idade) e um questionário abordando o principal estilo do nado, tempo de prática do esporte e tipo de respiração predominante. Na avaliação postural, utilizou-se uma câmera fotográfica digital Sony DSC-W 130 Cyber-Shot ${ }^{\circledR}$ e tripé WT3510A ${ }^{\circledR}$.

Após o preenchimento da ficha de identificação e do questionário, foi realizada a avaliação postural em vistas: anterior, posterior, perfil direito e perfil esquerdo. As fotos foram obtidas em sala isolada, com os atletas usando roupas adequadas para o exame, como short e top para o sexo feminino e short para o sexo masculino. A máquina fotográfica foi posicionada em um tripé a uma distância de $4 \mathrm{~m}$, e essa avaliação foi realizada por um fisioterapeuta qualificado, estando ele presente durante toda a coleta de dados.

\section{Avaliação postural}

1) Na vista anterior:

- foram demarcados os acrômios e as cristas ilíacas direita e esquerda;
- o atleta foi posicionado em ortostatismo, de frente para o fisioterapeuta, com a parte posterior do corpo voltada ao fio de prumo, com os pés ligeiramente afastados e rodados externamente $30^{\circ}$.

2) Na vista lateral:

- o atleta foi posicionado em ortostatismo de lado para o fisioterapeuta com o posicionamento do pé a $30^{\circ}$ (rotação lateral a partir da articulação de quadril);

- foram demarcados os seguintes pontos anatômicos: tubérculo maior, ápice da curvatura lombar, crista ilíaca;

- na vista lateral, o ponto de referência fixo é levemente anterior ao maléolo externo e representa o ponto básico do plano médio-coronal do corpo em alinhamento ideal.

3) Na vista posterior:

- o atleta posicionado em ortostatismo, de costas para o fisioterapeuta com o posicionamento do pé a $30^{\circ}$ (rotação lateral a partir da articulação de quadril);

- foram demarcados os seguintes pontos: ângulo inferior das escápulas e espinhas ilíacas póstero-superiores direita e esquerda.

- na vista posterior, a linha de referência vertical, divide o corpo em secções direita e esquerda;

- o ponto de referência fica a meio caminho entre os calcanhares e representa o ponto básico do plano médio sagital do corpo em alinhamento ideal;

- a análise estatística realizada foi exploratória descritiva, sendo utilizado o Teste Exato de Fisher para verificar a associação entre o tipo de respiração e as alterações posturais dos nadadores. Este procedimento foi realizado por meio do software $\mathrm{SAS}^{\circledR}$ 9, utilizando a PROC FREQ.

\section{Resultados}

Participaram deste estudo 60 nadadores de elite da equipe de natação da Unaerp, sendo 29 (48\%) mulheres e 31 (52\%) homens. $0 \mathrm{crawl}$ foi o estilo de nado predominante entre os atletas. Sobre o tipo 
de respiração, notamos que os homens respiravam mais do lado direito (49\%), e as mulheres (41\%), mais para o lado esquerdo. As características dos participantes estão descritas na Tabela 1.

Em relação às alterações posturais dos atletas, observamos diferenças significativas na região lombar, onde os homens apresentaram mais hiperlordose lombar (58\%) e as mulheres (31\%) escoliose convexa à direita $(\mathrm{p}=0,01)$, conforme a Tabela 2 .

As Tabelas 3 e 4 mostram os resultados que apresentaram associação entre a presença de alterações posturais e o tipo de respiração nos homens nadadores. Observamos que a maioria dos homens adotou a respiração do tipo unilateral à direita pelo fato de terem uma maior facilidade para realizar esse movimento e perderem menos tempo durante a respiração.

Durante a respiração à direita, o corpo do atleta realiza os seguintes movimentos: rotação do tronco à direita, uma leve flexão lateral do tronco à direita e uma rotação lateral da cabeça também à direita, tudo isso para dividir o gasto energético, tornando a exigência muscular menor.

Acreditamos que, pelo fato de o atleta respirar somente para a direita, poderá ter um desequilíbrio muscular, tornando a musculatura agonista desses movimentos mais forte do que a musculatura do lado contra lateral. Isso faz com que o atleta adquira desvios posturais como elevação do ombro esquerdo e uma leve escoliose convexa à esquerda asso-

Tabela 1 - Características dos participantes do estudo $(n=60)$

\begin{tabular}{lcc}
\hline & Homens & Mulheres \\
\hline Idade (anos) & $19,2 \pm 2,7$ & $19,8 \pm 3,2$ \\
Tempo de prática da natação (anos) & $7,5 \pm 3,4$ & $11,1 \pm 3,8$ \\
\hline Tipo de respiração dominante & $\mathbf{N}(\%)$ & $\mathbf{N}(\%)$ \\
\hline Unilateral à direita & $15(49)$ & $07(24)$ \\
Unilateral à esquerda & $06(19)$ & $12(41)$ \\
Bilateral & $10(32)$ & $10(35)$ \\
\hline Estilo do nado & $\mathbf{N}(\%)$ & $\mathbf{N ~ ( \% )}$ \\
\hline Crawl & $13(42)$ & $13(45)$ \\
Borboleta & $11(36)$ & $04(14)$ \\
Costas & $05(16)$ & $07(24)$ \\
Peito & $02(6)$ & $05(17)$ \\
\hline
\end{tabular}

Fonte: Dados da pesquisa. ciada à rotação da cintura escapular à direita (por causa do movimento combinado do tronco para realizar a respiração).

Embora não tenham apresentado diferenças significativas, notamos também que as mulheres que respiravam para o lado esquerdo apresentavam escoliose convexa à direita na coluna lombar (60\%), hipercifose torácica (80\%) e rotação da cintura pélvica à direita (40\%), enquanto os homens tinham rotação da cintura escapular à esquerda (67\%).

Tabela 2 - Prevalência de alterações posturais em atletas de natação $(n=60)$

\begin{tabular}{|c|c|c|c|}
\hline Alterações posturais & $\begin{array}{c}\text { Homens } \\
\text { N(\%) }\end{array}$ & $\begin{array}{c}\text { Mulheres } \\
\text { N(\%) }\end{array}$ & $\mathrm{p}$-valor \\
\hline \multicolumn{4}{|l|}{ Ombro } \\
\hline Normal & $08(26)$ & $10(34)$ & \multirow{3}{*}{0,72} \\
\hline Direito elevado & $11(35)$ & $08(28)$ & \\
\hline Esquerdo elevado & $12(39)$ & $11(38)$ & \\
\hline \multicolumn{4}{|l|}{ Coluna torácica } \\
\hline Normal & $03(10)$ & $02(7)$ & \multirow{4}{*}{0,09} \\
\hline Hipercifose & $18(58)$ & $15(52)$ & \\
\hline Escoliose convexa à direita & $06(19)$ & $07(24)$ & \\
\hline Escoliose convexa à esquerda & $04(13)$ & $05(17)$ & \\
\hline \multicolumn{4}{|l|}{ Coluna lombar } \\
\hline Normal & $04(13)$ & $03(10)$ & \multirow{4}{*}{0,01} \\
\hline Hipercifose & $18(58)^{*}$ & $12(41)$ & \\
\hline Escoliose convexa à direita & $04(13)$ & $09(31)^{*}$ & \\
\hline Escoliose convexa à esquerda & $05(16)$ & $05(18)$ & \\
\hline \multicolumn{4}{|l|}{ Joelhos } \\
\hline Normal & $10(32)$ & $10(34)$ & \multirow{2}{*}{0,99} \\
\hline Hiperextensão & $21(68)$ & $19(66)$ & \\
\hline \multicolumn{4}{|l|}{ Cintura escapular } \\
\hline Normal & $10(32)$ & $04(14)$ & \multirow{3}{*}{0,24} \\
\hline Rotação à direita & $10(32)$ & $10(34)$ & \\
\hline Rotação à esquerda & $11(36)$ & $15(52)$ & \\
\hline \multicolumn{4}{|l|}{ Cintura pélvica } \\
\hline Normal & $19(62)$ & $12(41)$ & \multirow{3}{*}{0,34} \\
\hline Rotação à direita & $06(19)$ & $09(31)$ & \\
\hline Rotação à esquerda & $06(19)$ & $08(28)$ & \\
\hline
\end{tabular}

Legenda: * $=p<0,05$.

Fonte: Dados da pesquisa. 
Tabela 3 - Associação entre as alterações posturais e o tipo de respiração entre os atletas de natação $(n=60)$

\begin{tabular}{lllccc}
\hline & & \multicolumn{4}{c}{ Ombro - N (\%) } \\
\cline { 3 - 5 } Sexo & Respiração & Normal & Direito elevado & Esquerdo elevado & p-valor \\
\hline \multirow{3}{*}{ Feminino } & bilateral & $5(41)$ & $1(8)$ & $6(50)$ & 0,45 \\
& direita & $2(28,5)$ & $3(43)$ & $2(28,5)$ & $3(30)$ \\
\hline \multirow{3}{*}{ Masculino } & esquerda & $3(30)$ & $4(40)$ & $2(20)$ & 0,02 \\
& bilateral & $3(30)$ & $2(50)$ & $10(67)^{*}$ & - \\
\hline
\end{tabular}

Legenda: * $=p<0,05$.

Fonte: Dados da pesquisa.

Tabela 4 - Associação entre as alterações posturais e 0 tipo de respiração entre os atletas de natação $(n=60)$

\begin{tabular}{lllcll}
\hline \multirow{2}{*}{ Sexo } & & \multicolumn{3}{c}{ Coluna torácica - N (\%) } & \multirow{2}{*}{-valor } \\
\cline { 3 - 5 } & Respiração & Escoliose convexa à direita & Escoliose convexa à esquerda & Normal & \\
\multirow{3}{*}{ Feminino } & bilateral & $4(33)$ & $5(42)$ & $3(25)$ & \\
& direita & $2(28,5)$ & $3(43)$ & $2(28,5)$ & 0,43 \\
& esquerda & $7(70)$ & $2(20)$ & $1(10)$ & \\
\hline \multirow{3}{*}{ Masculino } & bilateral & $2(20)$ & $2(20)$ & $6(60)$ & \\
& direita & $2(13)$ & $10(67)^{*}$ & $3(20)$ & 0,02 \\
& esquerda & $2(33)$ & - & $4(67)$ & \\
\hline
\end{tabular}

Legenda: * $=p<0,05$.

Fonte: Dados da pesquisa.

Os homens que respiravam para o lado direito apresentavam escoliose convexa à esquerda na coluna lombar (33\%), hiperlordose lombar (73\%), hipercifose torácica $(87 \%)$ e rotação à esquerda da cintura pélvica (33\%). As mulheres apresentaram apenas hiperlordose lombar (57\%).

\section{Discussão}

Somos seres biologicamente diferentes; sendo assim, a padronização do que seria uma boa postura é difícil de ser estabelecida, pois existe uma dependência entre postura e individualidade determinada por uma relação particular das estruturas corporais. A melhor postura que deve ser adotada por um indivíduo é aquela que preenche todas as necessidades mecânicas do seu corpo e também que possibilita ao indivíduo manter uma posição ereta com o mínimo esforço muscular, opor-se contra as forças externas, ter equilíbrio na realização do movimento e lutar contra a ação da gravidade.

São as curvaturas fisiológicas existentes na coluna vertebral que sustentam o peso da cabeça e das demais estruturas da coluna. Essa é uma das razões pelas quais grande parte dos problemas relacionados com a postura atingem essa estrutura.

Em relação às alterações posturais, observamos diferenças significativas para as variáveis hiperlordose lombar nos homens e escoliose convexa à direita nas mulheres, enquanto que Fernandes et al. (11) encontraram diferenças estatisticamente significantes somente para as variáveis hipercifose torácica e hiperextensão do joelho nos nadadores do sexo masculino. 
No estudo de Dezan et al. (12), 58\% dos atletas de luta olímpica apresentaram hiperlordose lombar. A presença da hiperextensão de joelhos, concomitantemente com a hiperlordose lombar, foi observada em $68,7 \%$ das atletas. Segundo Kendall et al. (9), nos casos de hiperlordose lombar pode-se apresentar uma ligeira anteriorização da coluna cervical em decorrência de mecanismos compensatórios.

Foi verificada neste estudo uma alta proporção de escoliose tanto no grupo dos homens como das mulheres, em que, dos 31 homens avaliados, 19 apresentaram escoliose, sendo 6 com escoliose convexa à direita na região torácica e 4 à esquerda, 4 com escoliose convexa à direita na região lombar e 5 à esquerda. Das 29 mulheres, 26 apresentaram escoliose, sendo 7 com escoliose convexa à direita na região torácica e 5 à esquerda, 9 com escoliose convexa à direita na região lombar e 5 à esquerda. Esses dados vão ao encontro do estudo de Santos et al. (13), no qual 94,6\% dos judocas avaliados apresentaram escoliose, bem como ao estudo de Moreira et al. (14), em que todos os 12 jogadores de basquete avaliados apresentaram escoliose, sendo $67 \%$ com predomínio destro-convexo lombar.

0 estudo de Tanchev et al. (15) sobre escoliose em atletas de ginástica rítmica mostrou que 12\% das atletas pesquisadas apresentavam escoliose, um número muito alto se comparado com meninas normais, não atletas, da mesma idade, que apresentaram $1,1 \%$.

Knoplich (16) cita que a incidência de problemas posturais é muito maior no sexo feminino do que no masculino, contrapondo os resultados encontrados no trabalho de Pinto e Lopes (17), em que não ocorreram diferenças significativas entre os sexos. Neste trabalho, no que se refere à incidência das alterações posturais, podemos observar que, para as variáveis estudadas, as mulheres apresentaram maiores incidências de problemas posturais do que os homens.

De acordo com Sizer et al. (18), grande parte das alterações posturais é decorrente da característica dos gestos motores e da forma como o treinamento é feito, já que alterações mecânicas e fisiológicas estão diretamente relacionadas com a atividade que o indivíduo desempenha. Geralmente existe uma tendência de se concentrar o trabalho de sobrecarga nos grupos musculares mais solicitados (responsáveis pelos gestos atléticos), desconsiderando a ação destes sobre a musculatura profunda, que é responsável pela manutenção da postura.
Segundo Neto Júnior, Pastre e Monteiro (19), a preocupação com a postura e o equilíbrio muscular devem ter o mesmo grau de importância que o desenvolvimento das qualidades específicas para o alto desempenho, pois a postura e o equilíbrio muscular influenciam no rendimento do atleta e podem minimizar a incidência de lesões esportivas. Segundo Santos et al. (13), pesquisas realizadas com jogadores juvenis de handebol de ambos os sexos deixaram claro que os desportos assimétricos podem conduzir a desvios posturais.

A prática de esportes de alto nível acaba por determinar padrões corporais específicos, de acordo com a modalidade praticada, por meio de adaptações musculoesqueléticas. Segundo Neto Júnior, Pastre e Monteiro (19), a exposição a uma rotina intensa e específica de exercícios físicos, típicos de cada desporto, produz um resultado estético, independe da nacionalidade, da etnia e dos hábitos de vida, que se traduz em alterações posturais associadas à eficiência do gesto desportivo. No entanto, a longo prazo, podem evoluir para processos crônicos que limitam o indivíduo para a prática de atividades físicas, sendo que, ainda, o maior tempo de prática esportiva está associado a maiores ângulos de alterações posturais (20).

A respiração, quando corretamente praticada, induz o relaxamento (21) e auxilia no combate de doenças do aparelho respiratório, assim como do coração e do sistema circulatório (22). Também auxilia na normalização das alterações posturais (23).

Para Stichert (24), o organismo que pratica respiração bilateral será suprido de mais oxigênio durante a inspiração seguinte, e terá mais tempo para a expiração. Mesmo com todas as vantagens da respiração bilateral, observamos no nosso estudo que os homens adotaram a respiração unilateral à direita e as mulheres, unilateral à esquerda. Esses resultados discordam da opinião de Machado (8) de que a respiração unilateral não deve ser a única ensinada durante a aprendizagem, pois o atleta pode aprender a respirar de um lado, passando sua vida atlética respirando somente desse lado e, dessa forma, pode desenvolver assimetricamente sua musculatura. Reforçando ainda a opinião desse autor, Gomes (25) enfatiza que em esportes praticados de forma unilateral pode ocorrer um desequilíbrio no desenvolvimento muscular. Por outro lado, Heltz (26) afirma que durante a aprendizagem deve-se induzir o aluno a respirar algumas vezes para o lado direito primeiramente e outras para o lado esquerdo e, a 
partir daí, o aluno deve escolher um dos lados que lhe agradar mais e respirar somente daquele lado.

Gonçalves et al. (27) afirmaram que atividades cíclicas e repetitivas, unidas com sobrecarga de treinamento, assim como a execução errada da técnica das braçadas e rotação unilateral para respiração, são fatores que contribuem para o desenvolvimento desigual da musculatura torácica, iniciando, assim, uma adaptação desigual que pode contribuir para o surgimento de uma escoliose. Esse desvio lateral da coluna vertebral foi observado nesta pesquisa em 19 homens e 26 mulheres atletas.

No nosso estudo observamos que 39\% dos homens e $38 \%$ das mulheres apresentaram ombro esquerdo elevado. De acordo com Pol et al. (28), o desnivelamento de ombro pode ser ocasionado pelo fato da lateralidade do atleta ser maior para a direita ou para a esquerda provocando esse desnível. De acordo com esses autores, o atleta exige muito de um dos lados do corpo e debilita a musculatura contrária.

Gonçalves et al. (27) enfatiza que a lateralidade representa uma força que proporciona desenvolvimento muscular desigual, confirmando por meio de estudos eletromiográficos que demonstram que durante o nado, principalmente no estilo crawl, os nadadores produzem grande pico de força com um dos braços, durante toda a fase de propulsão, lado esse correspondente ao dominante.

A prática esportiva tem se iniciado cada vez mais cedo, o que pode gerar alterações posturais dos atletas decorrentes do treinamento precoce, uma vez que o organismo das crianças ainda está em fase de desenvolvimento, sendo mais suscetíveis a sobrecargas externas (29).

O diagnóstico precoce das alterações posturais e a adoção de medidas profiláticas efetivas podem prevenir a ocorrência de lesões e desvios posturais na área desportiva, bem como, contribuir para o aumento do desempenho do atleta. Com o passar do tempo, o impacto de procedimentos de intervenção adequados, em diferentes períodos de treinamento do desportista, contribui para o aumento de sua qualidade de vida, minimizando, inclusive, o efeito de processos crônicos adquiridos com o trabalho corporal de alta intensidade (30).

Os estudos relacionados à prática da natação são poucos e não possuem uma correlação entre si. Não foram encontrados muitos artigos específicos relacionando alterações posturais com a prática da natação.

\section{Considerações finais}

Os resultados desta pesquisa revelaram que as mulheres apresentam mais alterações posturais e respiram mais para o lado esquerdo, porém, não se pode afirmar que a respiração influencia a postura dos atletas de natação. Por outro lado, a insuficiência de trabalhos publicados sobre a avaliação postural associada ao tipo de respiração de nadadores reforça a necessidade de novos estudos.

Acredita-se, assim, que a intervenção concomitante da Fisioterapia a fim de realizar um trabalho focado em reduzir a probabilidade de alterações posturais estruturais irreversíveis a longo prazo possa ser um meio profilático importante junto à prática esportiva da natação.

\section{Referências}

1. Mansoldo AC, Nobre DPA. Avaliação postural em nadadores federados praticantes do nado borboleta nas provas de 100 e 200 metros. Mundo da saúde. 2007; 31(4):511-20.

2. Schwartzmann NS, Santos FC, Bernardinelli E. Dor no ombro em nadadores de alto rendimento: possíveis intervenções fisioterapêuticas. Rev Ciênc Méd. 2005;14(2):199-212.

3. Bak K. Nontraumatic glenohumeral instability and coracoacromial impingement in swimmers. Scand J Med Sci Sports. 1996;6(3):132-44.

4. Garoff G, Catteau R. O ensino da natação. São Paulo: Manole; 1990.

5. Cabral F, Cristianni S, Souza WA. Natação 100 exercícios. Rio de Janeiro: Sprint; 1995.

6. Brasilone NJ. Natação: a didática da aprendizagem. Rio de Janeiro: Científica; 1995.

7. Thomas DG. Natação: etapas para o sucesso. São Paulo: Manole; 1999.

8. Machado DC. Natação: teoria e prática. Rio de Janeiro: Sprint; 1995.

9. Kendall FP, McCreary EK, Provance PG. Músculos provas e funções. São Paulo: Manole; 1995.

10. Bak K, Fauno P. Clinical findings in swimmers with shoulder pain. Am J Sports Med. 1997;25(2):254-60. 
11. Fernandes E, Mochizuki L, Amadio AC. Análise comparativa dos métodos de avaliação postural. Anais do II Congresso de Educação Física da Escola de Educação Física da Universidade de São Paulo, São Paulo; 1996. p. 15-21.

12. Dezan VH, Sarraf TA, Rodacki ALF. Aterações posturais, desequilíbrios musculares e lombalgias em atletas de luta olímpica. Rev Bras Ci e Mov. 2004;12(1): 35-8.

13. Santos JB, Michels G, Moro RP. Avaliação postural em atletas. Reabilitar. 2002;4(17):26-31.

14. Moreira PHC. Avaliação postural da seleção brasileira masculina de basquete. FisioBrasil. 2007;5:202-8.

15. Tanchev PI, Dzherov AD, Parushev AD, Dikov DM, Todorov MB. Scoliosis in Rhythmic Gymnasts. Spine. 2000;25(11):1367-72.

16. Knoplich J. Endireite as costas. São Paulo: Ibrasa; 1989.

17. Pinto HC, Lopes RF. Problemas posturais em alunos do centro de ensino médio 01 Paraná - Brasília DF. EFDeportes.com. 2001;7(42). [acesso 20 nov. 2010]. Disponível em: http://www.efdeportes.com.

18. Sizer RPS, Cook C, Brismée JM, Debrick L, Phelps V. Ergonomic pain - Part 1: etiology, epidemiology and prevention. Pain Pract. 2004;4(1):42-53.

19. Neto J Jr, Pastre CM, Monteiro HL. Alterações posturais em atletas brasileiros do sexo masculino que participaram de provas de potência muscular em competições internacionais. Rev Bras Med Esporte. 2004; 10(3):195-8.

20. Wojtys EM, Aashton-Miller JA, Huston LJ, Moga PJ. The association between athletic training time and the sagittal curvature of the immature spine. Am J Sports Med. 2000;28(4):490-8.
21. Campion MR. Hidroterapia: princípios e prática. São Paulo: Manole; 2000.

22. Massaud MG, Corrêa CRF. Natação para adultos. Rio de Janeiro: Sprint; 2001.

23. Kerbey FC. Natação: algo mais que 4 nados. São Paulo: Manole; 2002.

24. Stichert KH. Natação. Rio de Janeiro: Ao Livro Técnico; 1978.

25. Gomes SBS. O padrão postural estático em atletas da ULBRA. Rev Bras Ciênc Mov. 1995;16:11-5.

26. Heltz G. Natação: técnicas de aprendizagem e aperfeiçoamento. Munique: Europa-América; 1974.

27. Gonçalves DV, Santos ARB, Duarte CR, Matsudo VKR. Avaliação postural em praticantes de natação: uma análise critica. Rev Bras Ciênci Mov. 1989;3:16-23.

28. Pol DOC, Oliveira V, Wagner EM. Consequências do treinamento físico sobre a estrutura e postura de nadadores. Sprint. 1988;40:24-27.

29. Akachi PMH. A influência do treinamento competitivo do futsal na postura de atletas entre 9 e 16 anos. Rev Fisioter. 2001;8(2):97.

30. Braccialli LMP, Vilarta R. Aspectos a serem considerados na elaboração de programas de prevenção e orientação de problemas posturais. Rev Paulista de Educ Física. 2000;14:159-71.

Recebido: 09/10/2010 Received: 10/09/2010

Aprovado: 24/05/2011 Approved: 05/24/2011 